\title{
Postoperative nausea and vomiting: pharmacologic and nonpharmacologic therapies
}

\author{
Soo Kyung Lee \\ Department of Anesthesiology and Pain Medicine, Hallym University College of Medicine, Anyang, Korea
}

Postoperative nausea and vomiting (PONV) is the one of the most distressing problems associated with anesthesia and surgery. It may be cause dehydration, electrolyte imbalance, tension on the surgical repair site, venous hypertension and increased perception of pain, and may be especially harmful after neurosurgery or orthognathic surgery [1]. Many patients state that $\mathrm{PONV}$ is a more undesirable postoperative outcome than incisional pain [2].

PONV prophylaxis should be considered for patients at moderate to high risk for PONV, and antiemetic combination therapy or a multimodal approach are recommended for patients at high risk for PONV $[3,4]$. The concept of an antiemetic combination therapy was first introduced in 1988 by Parikh et al. [5] for chemotherapy induced vomiting. Prophylaxis with antiemetic drugs is the current gold standard for managing PONV. However, there is no completely effective pharmacologic agent for the prevention of PONV, and the potential adverse effects of antiemetic agents should not be overlooked. Nonpharmacologic methods such as acupuncture may also be used to as alternative or adjunct therapies to manage PONV. Dundee et al. [6] introduced the use of acupuncture as an antiemetic to manage PONV to the West in 1986. Although there was some debate over its effectiveness, many clinical studies were performed. A Cohrane review of 40 trials involving 4858 participants showed that P6 acupoint stimulation significantly reduced the incidence of PONV and the need for rescue antiemetics compared with sham treatment [7]. There are various methods of acustimulation in the prevention of PONV, such as manual [6] or electrical $[8,9]$ stimulation. It is reported that prevention of PONV by electri- cal acustimulation is a cheap and simple technique comparable to ondansetron during and after cesarean delivery under spinal anesthesia [8].

An alternative method to acupoint stimulation using a capsicum component has also been developed. Several studies have shown that the application of medical and pharmaceutical products containing capsicum component (e.g., capsicum plaster or ointment) at acupoints reduced the incidence of PONV [10-13]. Capsicum plaster at either the Korean hand acupuncture point K-D2 or the Pericardium 6 acupoint reduced PONV in patients undergoing abdominal hysterectomy [11]. Capsicum plaster at Hegu acupoints reduced the incidence of PONV and also conferred a postoperative analgesic effect after orthognathic surgery [12]. Also, the incidence of PONV and rescue anti-emetic requirement following laparoscopic cholecystectomy were reduced in patients who had capsaicin ointment applied to the K-D2 point [13].

In this issue of the Korean Journal of Anesthesiology [14], the authors found that applying capsicum plaster at the P6 or K-D2 acupoints more effectively lowered the incidence of PONV and the severity of nausea and improved satisfaction during the 24hr period after thyroid surgery, as compared to the control and sham groups. The incidence of nausea and vomiting during the $24 \mathrm{hr}$ after surgery was $24 \%$ and $4 \%, 22 \%$ and $6 \%, 59 \%$ and $30 \%$, and $50 \%$ and $24 \%$ in the P6, K-D2, control, and sham groups, respectively. Although the sample size of this study was not sufficiently large, it was calculated by a power analysis and the factors affecting PONV were comparable among the groups.

Several clinical studies have shown that pharmacologic and

Corresponding author: Soo Kyung Lee, M.D., Department of Anesthesiology and Pain Medicine, Hallym University College of Medicine, 22, Gwanpyeong-ro, 170 beon-gil, Dongan-gu, Anyang 431-796, Korea. Tel: 82-31-380-3945, Fax: 82-31-385-3244, E-mail: agnetask@yahoo.co.kr (c) This is an open-access article distributed under the terms of the Creative Commons Attribution Non-Commercial License (http:// creativecommons.org/licenses/by-nc/3.0/), which permits unrestricted non-commercial use, distribution, and reproduction in any medium, provided the original work is properly cited. 
nonpharmacologic combination therapies more effectively prevent PONV than pharmacologic therapy alone $[9,10]$. The combination of capsicum plaster at the Korean hand acupuncture points K-D2 with prophylactic ramosetron more effectively reduced the PONV compared with ramosetron alone in patients at high risk for PONV undergoing gynecologic laparoscopic surgery [10]. It is reported that perioperative P6 acustimulation with transcutaneous electrical acupoint stimulation may be an effective adjunct to the standard antiemetic drug therapy with ondansetron and dexamethasone to prevent PONV in patients undergoing infratentorial craniotomy [9].

The incidence of PONV is still high in some patients. Acupuncture and related therapies may be used as inexpensive and effective alternative or adjunct therapies along with standard antiemetic therapy for preventing PONV. Further studies are required to identify the optimal timing and effective method of acustimulation and combination with pharmacologic antiemetic therapies.

\section{References}

1. Watcha MF, White PF. Postoperative nausea and vomiting. Its etiology, treatment, and prevention. Anesthesiology 1992; 77: 162-84.

2. Macario A, Weinger M, Carney S, Kim A. Which clinical anesthesia outcomes are important to avoid? The perspective of patients. Anesth Analg 1999; 89: 652-8.

3. Gan TJ, Meyer T, Apfel CC, Chung F, Davis PJ, Eubanks S, et al. Consensus guidelines for managing postoperative nausea and vomiting. Anesth Analg 2003; 97: 62-71.

4. Gan TJ, Meyer TA, Apfel CC, Chung F, Davis PJ, Habib AS, et al. Society for Ambulatory Anesthesia guidelines for the management of postoperative nausea and vomiting. Anesth Analg 2007; 105: 1615-28.

5. Parikh PM, Charak BS, Banavali SD, Koppikar SB, Giri N, Nadkarni P, et al. A prospective, randomized double-blind trial comparing metoclopramide alone with metoclopramide plus dexamethasone in preventing emesis induced by high-dose cisplatin. Cancer 1988; 62: 2263-6.

6. Dundee JW, Chestnutt WN, Ghaly RG, Lynas AG. Traditional Chinese acupuncture: a potentially useful antiemetic? Br Med J (Clin Res Ed) 1986; 293: 583-4.

7. Lee A, Fan LT. Stimulation of the wrist acupuncture point P6 for preventing postoperative nausea and vomiting. Cochrane Database Syst Rev 2009; (2): CD003281.

8. El-Deeb AM, Ahmady MS. Effect of acupuncture on nausea and/or vomiting during and after cesarean section in comparison with ondansetron. J Anesth 2011; 25: 698-703.

9. Xu MX, Zhou SJ, Jiang CC, Wu Y, Shi WL, Gu HH, et al. The effects of P6 electrical acustimulation on postoperative nausea and vomiting in patients after infratentorial craniotomy. J Neurosurg Anesthesiol 2012; 24: 312-6.

10. Jung HJ, Park SY. Combination of capsicum plaster at the Korean hand acupuncture points K-D2 with prophylactic antiemetic on postoperative nausea and vomiting after gynecologic laparoscopy. J Korean Acad Nurs 2013; 43: 215-24.

11. Kim KS, Koo MS, Jeon JW, Park HS, Seung IS. Capsicum plaster at the korean hand acupuncture point reduces postoperative nausea and vomiting after abdominal hysterectomy. Anesth Analg 2002; 95: 1103-7.

12. Kim KS, Kim KN, Hwang KG, Park CJ. Capsicum plaster at the Hegu point reduces postoperative analgesic requirement after orthognathic surgery. Anesth Analg 2009; 108: 992-6.

13. Agarwal A, Dhiraaj S, Tandon M, Singh PK, Singh U, Pawar S. Evaluation of capsaicin ointment at the Korean hand acupressure point K-D2 for prevention of postoperative nausea and vomiting. Anesthesia 2005; 60: 1185-8.

14. Koo MS, Kim KS, Lee HJ, Jeong JS, Lee JW. Antiemetic efficacy of capsicum plaster on acupuncture points in patients undergoing thyroid operation. Korean J Anesthesiol 2013; 65: 539-43. 